Nordic Machine Intelligence, MedAI 2021

https://doi.org/10.5617/nmi.9157

\title{
Attention U-Net ensemble for interpretable polyp and instrument segmentation
}

\author{
Michael Yeung ${ }^{1,2}$ \\ 1. School of Clinical Medicine, University of Cambridge, Cambridge, UK \\ 2. Department of Radiology, University of Cambridge, Cambridge, UK
}

\begin{abstract}
The difficulty associated with screening and treating colorectal polyps alongside other gastrointestinal pathology presents an opportunity to incorporate computer-aided systems. This paper develops a deep learning pipeline that accurately segments colorectal polyps and various instruments used during endoscopic procedures. To improve transparency, we leverage the Attention U-Net architecture, enabling visualisation of the attention coefficients to identify salient regions. Moreover, we improve performance by incorporating transfer learning using a pre-trained encoder, together with test-time augmentation, softmax averaging, softmax thresholding and connected component labelling to further refine predictions.
\end{abstract}

Keywords: artificial intelligence; machine learning; segmentation; transparency; medicine

\begin{abstract}
Introduction
The Norwegian Artificial Intelligence Research Consortium (NORA) MedAl challenge aims to encourage the development of automatic medical image segmentation systems that are both accurate and explainable [1]. The challenge involves developing a pipeline to perform colorectal polyp and endoscopic instrument segmentation, favouring transparent and explainable solutions. To achieve both aims, we develop a deep learning pipeline that leverages the Attention U-Net for transparency, together with several post-processing stages to refine predictions: test-time augmentation, softmax averaging, softmax thresholding and connected component labelling.
\end{abstract}

\section{Methods}

Attention U-Net

Currently, the most widely used convolutional neural network (CNN) for medical image segmentation is U-Net [2]. The Attention U-Net incorporates Attention Gates (AGs) into U-Net, which uses a gating signal that aggregates multiscale spatial information to highlight salient features present in skip connections [3]. Importantly, attention coefficients provide interpretable intermediate outputs at each depth, and it has been observed that the AGs learn during training to localise target regions. We leverage the interpretable nature of the Attention U-Net, with the performance benefits of transfer learning, combining a ResNet152 encoder pre-trained on ImageNet with an Attention U-Net decoder network [4]. The network architecture is shown in Figure 1.

\section{Prediction pipeline}

To further improve accuracy, we perform a series of postprocessing steps on the network predictions. The full prediction pipeline is shown in Figure 2.

Firstly, we perform test-time augmentation using a series of $90^{\circ}$ rotations and reflections to generate 8 variants for each input image, and average the resulting predictions [5]. Next, we incorporate ensemble learning by averaging the softmax outputs generated by each network [6]. To control the recall-precision bias, the ensembled predictions for the segmentation target are compared to a threshold, assigning the segmentation target class to a pixel if above the threshold.

Finally, after resizing the segmentation labels to the original resolution, we use connected component labelling to identify and remove any regions assigned to the segmentation target occupying $<1 \%$ of the image, reducing the false positive predictions and generating the final prediction.

\section{Dataset descriptions and evaluation metrics}

Two training datasets were provided for this challenge: the Kvasir-SEG dataset for polyp segmentation and the Kvasir-Instrument dataset for endoscopic instrument segmentation $[7,8]$. For evaluation, the Dice Similarity 


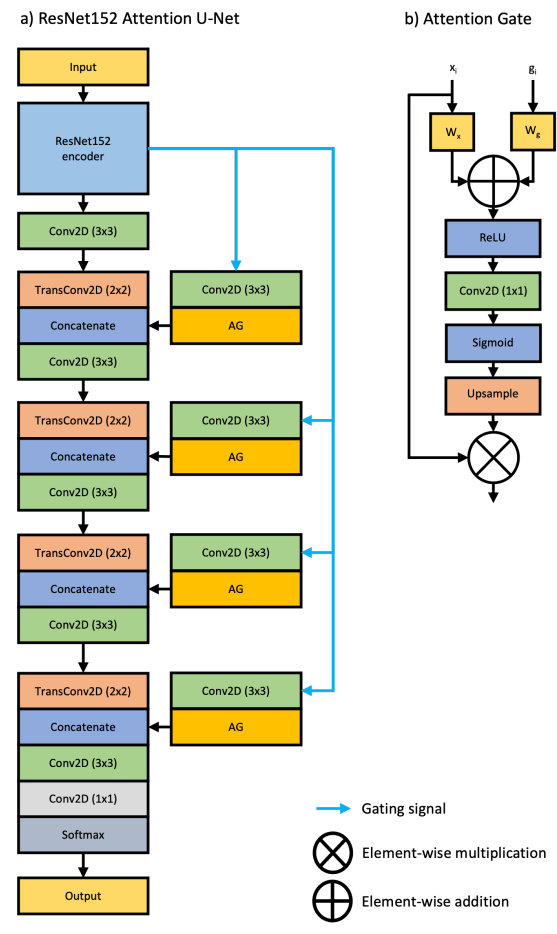

Figure 1: a) ResNet152 Attention U-Net network architecture. Each $3 \times 3$ convolution is followed by instance normalisation and ReLU activation. b) Attention Gate. The gating signal $\left(W_{g}\right)$ uses contextual information to modify incoming skip connections $\left(W_{x}\right)$ at each level.

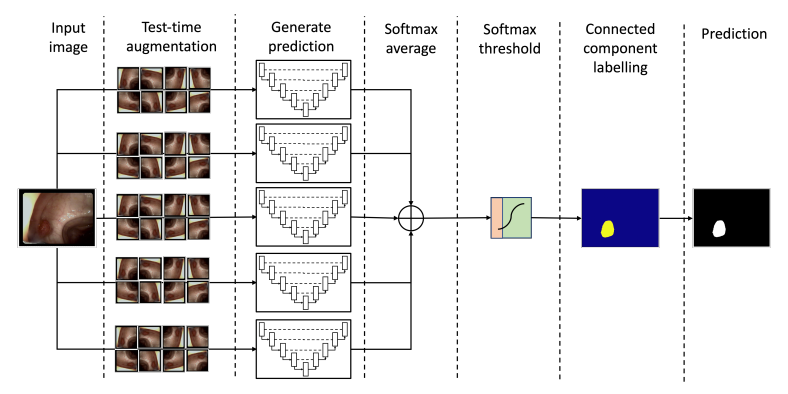

Figure 2: Overview of the prediction pipeline. The query image is transformed using test-time augmentation, and individually generated softmax outputs are then averaged and thresholded. Once resized, connected component labelling identifies and removes any regions occupying $<1 \%$ of the image.

Coefficient (DSC), Jaccard index, accuracy, precision and recall metrics were calculated for each image and averaged over the hold-out test set.

\section{Implementation details}

Our experiments were programmed using Keras with Tensorflow Backend, and made use of the Medical Image Segmentation with CNN (MIScnn) open-source Python library [9]. Models were trained using NVIDIA P100 GPUs. For each dataset, we randomly partition images into five folds consisting of $80 \%$ training set and $20 \%$
Table 1: Performance comparisons on the hold-out test set using different softmax thresholds.

\begin{tabular}{l|cccccc}
\hline Target & Threshold & Jaccard & Dice & Recall & Precision & Accuracy \\
\hline \multirow{5}{*}{ Polyp } & 0.50 & 0.7910 & 0.8606 & $\mathbf{0 . 8 9 9 4}$ & 0.8632 & 0.9539 \\
& 0.45 & 0.7983 & 0.8649 & 0.8940 & 0.8734 & 0.9586 \\
& 0.40 & 0.8033 & 0.8683 & 0.8885 & 0.8838 & 0.9619 \\
& 0.35 & 0.8080 & $\mathbf{0 . 8 7 1 6}$ & 0.8815 & 0.8960 & 0.9645 \\
& 0.30 & $\mathbf{0 . 8 0 8 3}$ & 0.8701 & 0.8702 & $\mathbf{0 . 9 0 5 2}$ & $\mathbf{0 . 9 6 6 3}$ \\
\hline \multirow{5}{*}{ Instrument } & 0.50 & 0.9023 & 0.9412 & $\mathbf{0 . 9 5 9 2}$ & 0.9352 & 0.9838 \\
& 0.45 & 0.9056 & 0.9429 & 0.9561 & 0.9414 & 0.9856 \\
& 0.40 & 0.9079 & $\mathbf{0 . 9 4 4 2}$ & 0.9527 & 0.9468 & 0.9871 \\
& 0.35 & $\mathbf{0 . 9 0 8 5}$ & 0.9437 & 0.9454 & 0.9514 & 0.9883 \\
& 0.30 & 0.9048 & 0.9397 & 0.9374 & $\mathbf{0 . 9 5 2 3}$ & $\mathbf{0 . 9 8 9 3}$ \\
\hline
\end{tabular}

validation set, training five models using separate dataset folds. All images were resized to $512 \times 512$ pixels and normalised to $[0,1]$ using the $z$-score. During training, we applied the following data augmentation: scaling, rotation, mirroring, elastic deformation and brightness.

Model parameters were initialised using the Xavier initialisation [10]. We trained each model with a batch size of 1 using the stochastic gradient descent optimiser with an initial learning rate of 0.1. We used ReduceLROnPlateau to reduce the learning rate by 0.1 if the validation loss did not improve after 25 epochs, and the EarlyStopping callback to terminate training if the validation loss did not improve after 75 epochs.

We optimised the models using the Unified Focal loss, a compound loss function designed to handle class imbalanced datasets [11]. We set $\delta=0.6$ and $\lambda=0.5$, and empirically determined the optimal $\gamma$ value for each dataset, setting $\gamma=0.3$ and $\gamma=0.2$ for Kvasir-SEG and Kvasir-Instrument respectively.

The source code is available at: https://github.com/ $\mathrm{mlyg} /$ nora-challenge-camai.

\section{Results}

The results are shown in Table 1. The highest Jaccard score was obtained with a softmax threshold at 0.30 and 0.35 for the polyp and instrument segmentation tasks respectively, correcting the original recall bias present.

\section{Discussion}

Our deep learning pipeline achieves accurate predictions for both polyp and instrument segmentation tasks, while remaining transparent and explainable. The softmax threshold may be adjusted to improve recall or precision, and here we have shown its usage to balance recall and precision to optimise the Jaccard and Dice values. Future work will focus on improving efficiency towards the aim of developing real-time segmentation systems.

\section{Conflict of interest}

Authors state no conflict of interest.

\section{Acknowledgements}

This work was performed using resources provided by the Cambridge Service for Data Driven Discovery (CSD3) operated by the University of Cambridge Research Computing Service (www.csd3.cam.ac.uk). 


\section{References}

1. Hicks $S$, Jha D, Thambawita $V$, Riegler M, Halvorsen $P$, Singstad B, Gaur S, Pettersen K, Goodwin M, Parasa S, and Lange $T$ de. MedAl: Transparency in Medical Image Segmentation. Nordic Machine Intelligence 2021. DOI: $10.5617 / \mathrm{nmi} .9140$

2. Ronneberger O, Fischer P, and Brox T. U-Net: Convolutional networks for biomedical image segmentation. Proc. International Conference on Medical Image Computing and Computer-Assisted Intervention (MICCAI). Springer. $2015: 234-41$

3. Schlemper J, Oktay O, Schaap M, Heinrich M, Kainz B, Glocker B, and Rueckert D. Attention gated networks: Learning to leverage salient regions in medical images. Med. Image Anal. 2019; 53:197-207

4. Jha D, Riegler MA, Johansen D, Halvorsen P, and Johansen HD. Doubleu-net: A deep convolutional neural network for medical image segmentation. 2020 IEEE 33rd International symposium on computer-based medical systems (CBMS). IEEE. 2020 :558-64

5. Jha $D$, Smedsrud $P H$, Johansen $D$, Lange $T$ de, Johansen HD, Halvorsen $P$, and Riegler MA. A comprehensive study on colorectal polyp segmentation with ResUNet++, conditional random field and test-time augmentation. IEEE journal of biomedical and health informatics 2021; 25:2029-40

6. Ju C, Bibaut $A$, and Laan $M$ van der. The relative performance of ensemble methods with deep convolutional neural networks for image classification. Journal of Applied Statistics 2018; 45:2800-18

7. Jha D, Smedsrud PH, Riegler MA, Halvorsen P, Lange $T$ de, Johansen $D$, and Johansen HD. Kvasir-SEG: A segmented polyp dataset. Proc. International Conference on Multimedia Modeling (MMM). Springer. 2020 :45162

8. Jha D, Ali S, Emanuelsen K, Hicks SA, Thambawita V, Garcia-Ceja E, Riegler MA, Lange T de, Schmidt PT, Johansen HD, et al. Kvasir-instrument: Diagnostic and therapeutic tool segmentation dataset in gastrointestinal endoscopy. International Conference on Multimedia Modeling. Springer. $2021: 218-29$

9. Müller D and Kramer F. MIScnn: a framework for medical image segmentation with convolutional neural networks and deep learning. BMC Med. Imaging 2021; 21:1-11

10. Glorot $X$ and Bengio $Y$. Understanding the difficulty of training deep feedforward neural networks. Proceedings of the thirteenth international conference on artificial intelligence and statistics. JMLR Workshop and Conference Proceedings. 2010 :249-56

11. Yeung M, Sala E, Schönlieb CB, and Rundo L. Unified Focal loss: Generalising Dice and cross entropy-based losses to handle class imbalanced medical image segmentation. arXiv preprint arXiv:2102.04525 2021 